\title{
EXIST: The Ultimate Spatial/Temporal Hard X-ray Survey
}

\author{
J. Grindlay ${ }^{1}$, L. Bildsten ${ }^{2}$, R. Blandford ${ }^{3}$, D. Chakrabarty 4 , M. Elvis ${ }^{1}$,
}

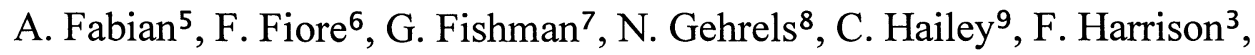
D. Hartmann ${ }^{10}$, C. Kouveliotou ${ }^{7}$, T. Prince ${ }^{3}$, B. Ramsey ${ }^{7}$, R. Rothschild ${ }^{11}$, G. Skinner ${ }^{12}$, and S. Woosley ${ }^{13}$

1. CfA, 2. UCSB, 3. Caltech, 4. MIT, 5. IoA, 6. Rome Obs., 7. MSFC, 8. GSFC, 9. Columbia Univ., 10., Clemson, 11. UCSD, 12. CESR, 13. UC Santa Cruz (EXIST Science Team institutions)

\begin{abstract}
The Energetic X-ray Imaging Survey Telescope (EXIST) is a proposed mission to conduct an all-sky imaging hard x-ray (HX) survey $(\sim 5-600 \mathrm{keV})$ with $\sim 0.05 \mathrm{mCrab}$ sensitivity $(5 \sigma ; 6 \mathrm{mo} . ; \sim 5-100 \mathrm{keV})$ comparable to the ROSAT soft $\mathrm{x}$-ray survey, and to provide the maximum sensitivity and resolution (spatial and temporal) HX imager as the Next Generation GRB mission. Its primary science goals are to i) identify and measure obscured AGN and constrain the accretion luminosity of the universe as well as the cosmic IR background from Blazar spectra coincident with $\mathrm{GeV}-\mathrm{TeV}$ observations, ii) measure spectra, variability and locations for the faintest GRBs to study the most energetic events in the universe and the earliest epoch of star formation, and iii) study black holes on all scales, from x-ray transients to luminous AGN. EXIST would incorporate a very large area $\left(\sim 8 \mathrm{~m}^{2}\right)$ imaging Cd-Zn-Te detector and coded aperture telescope array with nearly half-sky instantaneous view which images the full sky each orbit. With fixed zenith pointing, it could be mounted on the ISS or a free flyer and would complement both GLAST and Constellation-X science if launched before 2010, as recommended by the Astronomy and Astrophysics Decadal Survey.
\end{abstract}

\section{REVEALING THE OBSCURED HARD X-RAY UNIVERSE}

At energies $>5-10 \mathrm{keV}$, the buried nuclei of active galaxies, which provide most of the cosmic x-ray background, become increasingly clear (if not Compton thick) and reveal the accretion power of the universe [1]. The hard $\mathrm{x}$-ray (HX) band provides the most direct view of the central regions of AGN and near-horizon views of black holes; lower energies are often absorbed and $\gamma$-ray emission is likely converted to pairs in the most compact regions. The energy band from $\sim 5-600 \mathrm{keV}$, which covers the transition from the thermal $x$-ray universe of hot gas traced by ubiquitous Fe line emission (6.4$6.7 \mathrm{keV})$ to the extremes of pair plasmas $(511 \mathrm{keV})$ and the non-thermal universe, is rich in phenomena and yet remains relatively obscure in realization. The sky has not yet been surveyed in this band with the sensitivity or imaging resolution achieved in 1991 with ROSAT for the soft x-ray band. The last full-sky HX survey, carried out with HEAO-1 in 1979 [2] was sensitive to sources only down to $\sim 1 / 20$ of the brightest (Crab, CygX-1) for uncrowded high latititude fields, and had only coarse angular 
resolution $\left(\sim 3^{\circ}\right)$ and energy resolution $(\sim 20 \%)$. An imaging (17' resolution) HX survey with $\sim 10-30 \mathrm{X}$ the HEAO sensitivity will be conducted with the upcoming Swift mission [3] and over a similar energy band $(\sim 15-150 \mathrm{keV})$ as the HEAO-A4 survey.

The EXIST (Energetic X-ray Imaging Survey Telescope) mission, proposed first as a New Mission Concept in 1994 [4] and recently recommended for implementation within the current decade by the Astronomy/Astrophysics Decadal Review, would conduct the "ultimate" HX survey: it would extend the energy band to cover the full HX to soft $\gamma$-ray range $(\sim 5-600 \mathrm{keV})$ with the broadest possible temporal coverage (microsec to months) to measure the inherently variable HX sky in both space and time. Such broad energy and temporal coverage requires use of the coded aperture imaging technique (cf. Caroli et al [5]), whereby position-sensitive detectors record shadows of the distribution of sources cast by a coded mask and which then achieves sensitivity for minimum fluxes decreasing with total detector area $\mathrm{A}$, integration time $\mathrm{T}$ and recorded background $\mathrm{B}$ as $(T / A \cdot B)^{0.5}$. EXIST would extend the coded aperture technique to the practical limits, with total detector area $\mathrm{A} \sim 8 \mathrm{~m}^{2}$ and effective exposure time (per source) T maximized so that any source is observed for $>20 \%$ of the time and the full sky is imaged each $95 \mathrm{~min}$ orbit. This combination of very large area and field of view, and thus source temporal coverage, makes EXIST also the core of the "Next Generation Gamma-Ray Burst (GRB) Mission".

We present an overview of the EXIST mission concept and then describe the major scientific objectives that are made possible by the proposed detector-telescope configuration. The technical implementation of EXIST, as currently envisioned [6] for the International Space Station (ISS), is then outlined along with a brief description of the mission development plan. Studies for a free flyer implementation will be conducted, along with continuing programs for detector development and balloon flight tests.

\section{OVERVIEW OF EXIST CONCEPT}

The possible implementation of EXIST on the ISS is shown in Figure 1. Mounted on the P3 Attached Payloads site, EXIST is configured as two large-area ( $4 \mathrm{~m}^{2}$ each) and large field of view (FOV; $80^{\circ} \times 80^{\circ}$ each) coded aperture telescopes that are mounted back-to-back along the main truss of the ISS. The combined FOV ${ }^{1}$ of $160^{\circ} \mathrm{x}$ $80^{\circ}$ is aligned along the truss, or perpendicular to the orbital ram direction, so that the combined fan beam sweeps out the entire sky each $95 \mathrm{~min}$ orbit. The instantaneous FOV of almost $\sim 60 \%$ of the sky un-occulted by Earth gives good sensitivity and coverage for bursts and fast transients.

The primary driver for the detector and telescope design is the desired survey sensitivity: the science goals described below are based on a desired all-sky survey sensitivity of $0.05 \mathrm{mCrab}(5 \sigma ; 6$ months) over the $\sim 5-100 \mathrm{keV}$ band, yielding flux

${ }^{1}$ The EXIST-ISS concept is a contiguous $2 \times 4$ array of $40^{\circ}$ (FWHM) fields. Thus the response is flat over $120^{\circ} \times 40^{\circ}$, reduced to 0.5 at $160^{\circ} \times 80^{\circ}$ and 0.25 at $180^{\circ} \times 100^{\circ}$, yielding relative (sensitivities, half-sky fractions) of: $(1,0.23),(0.7,0.62),(0.5,0.88)$. 
sensitivities of $F_{X}=5 \times 10^{-13} \operatorname{cgs}(5-10 \mathrm{keV})$ or $9 \times 10^{-13} \operatorname{cgs}(20-100 \mathrm{keV})$. This matches the ROSAT all-sky soft $\mathrm{x}$-ray $(0.5-2.5 \mathrm{keV})$ survey sensitivity, allowing the first extension into the HX band of a survey able to detect and locate the same objects for a (typical) Crab-like spectrum. The corresponding optical magnitude for an object

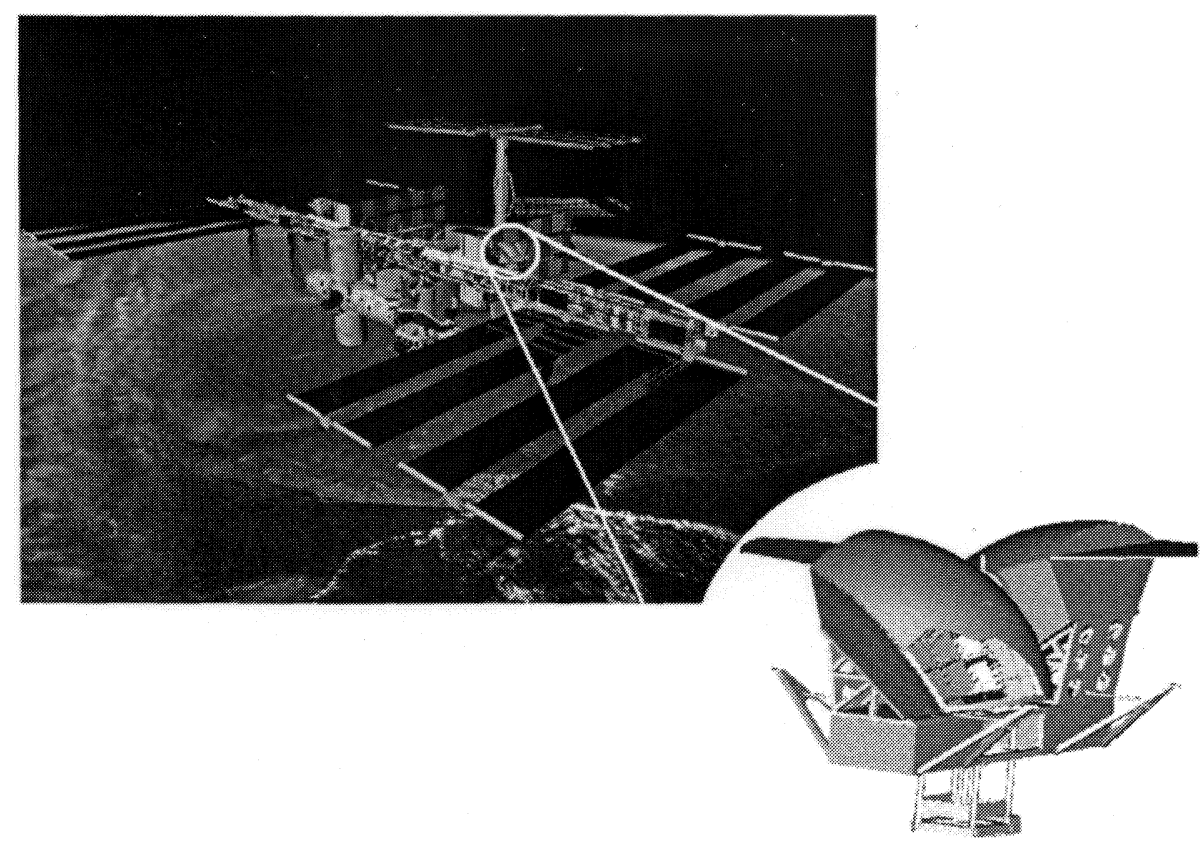

FIGURE 1. EXIST concept for ISS: back-to-back $80^{\circ} \times 80^{\circ}$ coded aperture telescopes image (5') the full sky each orbit. Each telescope contains $2 \times 2$ contiguous $1 \mathrm{~m}^{2}$ arrays of CZT imaging detectors (partly visible), each actively collimated to $40^{\circ} \times 40^{\circ}$, to view the sky through an extended hemispherical coded aperture.

with x-ray/optical flux ratio $F_{X} / F_{V}=1$ (such as for many CVs and AGN) is $\mathrm{V}=20.9$, using the relation between $F_{V}$ and $\mathrm{V}$ magnitude, $\log F_{V}=-3.96-0.4 \mathrm{~V}$, which combines the near-uv and optical flux. The sensitivity goal requires a large total detector area $\left(8 \mathrm{~m}^{2}\right)$, which is realized by $8 \times 1 \mathrm{~m}^{2}$ telescopes each with $40^{\circ} \times 40^{\circ}$ FOV (cf. Fig. 1).

The detectors are Cd-Zn-Te (CZT) crystals, each perhaps $20 \mathrm{~mm} \times 20 \mathrm{~mm}$ and 5 $10 \mathrm{~mm}$ thick, and fabricated with anode pixels $(1.3-2.5 \mathrm{~mm}$ pixel pitch) which contact onto a coupling board with VLSI/ASIC readout to record the peak pulse height pixel and nearest neighbors for each X-ray event. A part of the total CZT array $(\sim 1 / 4$ total area) may be apportioned to a low energy $(<100 \mathrm{keV})$ optimzed array $(0.6 \mathrm{~mm}$ pixels; $2 \mathrm{~mm}$ thick CZT) with reduced FOV $\left(10^{\circ} \times 40^{\circ}\right)$ to reduce bright source backgrounds. The CZT crystals could be grouped $2 \times 2$ onto a common ASIC board (cf. Fig. 8), which are then in turn close-tiled into a large area array (possibly $32 \mathrm{~cm} \times 32 \mathrm{~cm}$ ) with 
common digital readout and control as well as surrounding collimating shield $(1 \mathrm{~cm}$ CsI). Relatively thick CZT ( $\geq 5 \mathrm{~mm}$ ) is needed for the primary detector to extend the response to $600 \mathrm{keV}$, and depth sensing would be used to reduce detector background. For $5 \mathrm{~mm}$ thick CZT, the $\sim 100-600 \mathrm{keV}$ sensitivity is $\sim 0.5 \mathrm{mCrab}$ (cf. Fig. 2).

Source confusion is avoided at the projected sensitivity (cf. Fig. 2), which should detect $\geq 1 \mathrm{AGN} /$ square degree (see below), by imaging with $5^{\prime}$ resolution (a factor of 2 better than the usual criteria of $\sim 1 / 40$ source per "beam"). A source on the orbital equator is in the FOV for a fraction $80 / 360=0.22$ of the time, whereas those near the

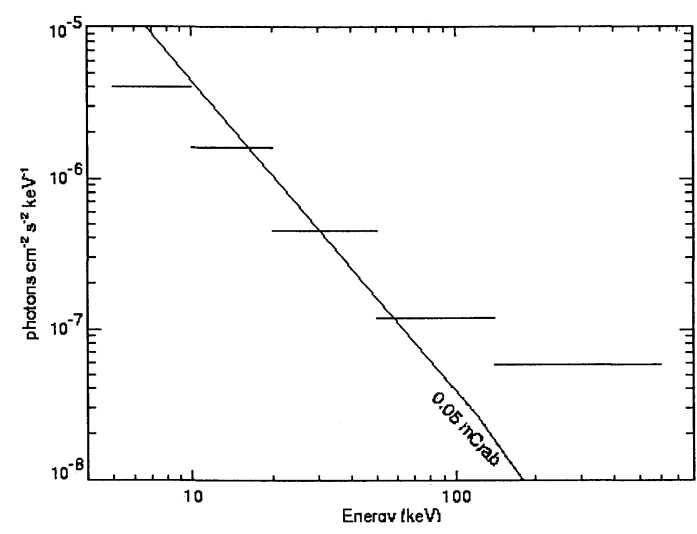

FIGURE 2. Approximate continuum sensitivity $(5 \sigma$, lyear) for EXIST-ISS configuration. Narrow line sensitivities (phot $/ \mathrm{cm}^{2}-\mathrm{sec}$ ) are reduced by factor of $\sim 10$. orbital poles are nearly continuously observed. The collimation provided by the active shields gives a FOV of $40^{\circ} \times 40^{\circ} \mathrm{FWHM}$ (cf. footnote 1) for each $1 \mathrm{~m}^{2}$ telescope is, so that in fact $1 / 4$ of the area of the telescopes is available at the $\pm 90^{\circ}$ limits of the combined FOV to cover the orbital poles. Likewise, in the scan direction the FOV is similarly extended from $80^{\circ}$ to $100^{\circ}$, at $1 / 4$ response, thereby increasing the total minimum source exposure fraction to $100 / 360=0.28$. Thus the full sky is imaged and monitored each $95 \mathrm{~min}$ orbit.

\section{EXIST SCIENTIFIC OBJECTIVES}

\section{Obscured AGN}

EXIST would achieve a sensitivity for AGN that would allow the $\log N-\log S$ distribution of obscured objects to be measured over the full sky for the first time. As pointed out by Fabian [7], the 3 closest AGN (Cen A, NGC 4945, and the Circinus galaxy) are all obscured. What is not known from optical surveys is how many more highly obscured Seyfert II galaxies are relatively nearby. These would be revealed by the EXIST survey and could then be studied in detail by followup observations with higher sensitivity and resolution. BeppoSAX spectra [8] of two optically selected and relatively nearby Seyfert II systems, NGC1068 and NGC6240, are shown in Figure 3 and are indicative of what the $\mathrm{x}$-ray selected survey sample might look like.

An unbiased sample of the number of obscured AGN is essential for testing models of the cosmic x-ray background and the likely contribution of obscured AGN. The relative contribution of obscured AGN (with $\log \mathrm{NH} \geq 23.5$ ) to the total number counts is predicted [9] to exceed that of unabsorbed AGN by factors of $\geq 2-4$ at flux levels of $\log \mathrm{F}(5-10 \mathrm{keV}) \sim-12.5$ (cgs), which is readily measured with EXIST. 


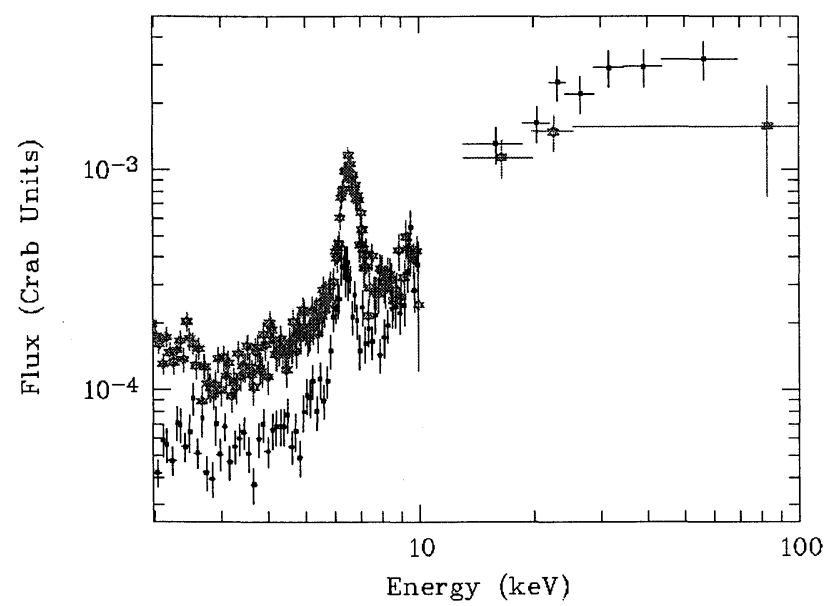

FIGURE 3. BeppoSAX spectra [8] of optically selected Seyfert II's: NGC1068 (top) \& NGC6240. EXIST would go 20X deeper, full sky.
With its sensitivity for AGN that are highly obscured (and becoming Compton thick), and with the overall AGN sample expected (from $\log \mathrm{N}-\log \mathrm{S}$ ) to be at least $\sim 30,000$, EXIST will provide a large sample of both nearby Seyfert II's and more distant and luminousType 2 QSOs. The luminosity and redshift distributions of the obscured AGN will measure the accretion vs. nuclear (starlight) power in the universe.

\section{Blazars and the Diffuse IR Background}

A related objective is to measure the intrinsic HX spectra of Blazars, highly beamed and variable AGN, in order that (near)-simultaneous $\gamma$-ray observations

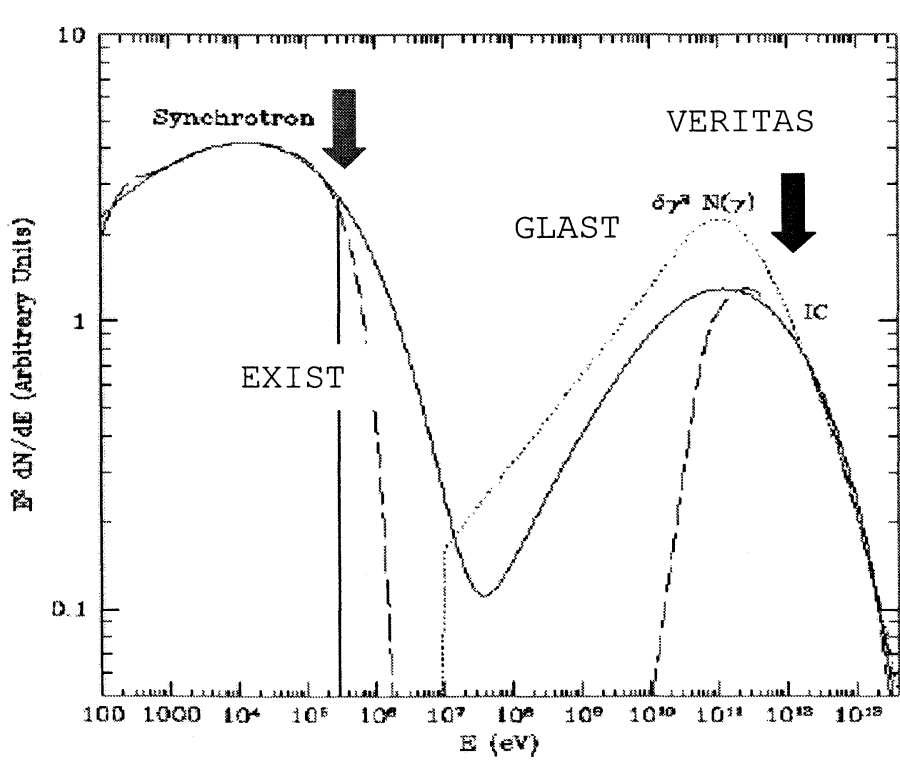

FIGURE 4. Adapted from [10]: HX spectral break (EXIST) constrains the GeV-TeV break (GLAST-VERITAS) and thus the cosmic diffuse IR. can be combined to constrain the diffuse IR background at $\sim 300 \mu$. The recent detections of several blazars as $\mathrm{TeV}$ sources (cf. Weekes, these proceedings) shows that these "extreme" BL Lac objects (e.g. Mkn 501) are detected at distances $(\mathrm{z} \sim 0.03)$ where their $\mathrm{TeV}$ photons could have undergone photonphoton absorption on the cosmic IR background photons (converting electron pairs). 
These "blue blazars" have spectral energy distributions (SEDs) with a broad peak in the $\mathrm{x}$-ray/hard $\mathrm{x}$-ray and a second peak in the GeV-TeV gamma-ray band (cf. Figure 4). The natural interpretation (e.g. Coppi \& Aharonian [10]), increasingly favored, is that the emission is synchrotron self-Compton (SSC). Thus a simultaneous measurement of the synchrotron spectral cutoff (likely to be time variable) with EXIST allows the observed inverse Compton TeV spectral break to constrain the diffuse IR flux. Interestingly, this IR background is likely produced in part by the same obscured AGN (and starburst galaxies) responsible for the HX background and directly detected by EXIST.

\section{Gamma-Ray Bursts}

The capability of EXIST for high sensitivity/resolution hard x-ray imaging over a wide field and energy band renders it particularly suitable for GRB science, which together with the HX survey objectives are the primary science drivers of the mission. Indeed, the study of GRBs at the faintest flux levels, as well as high time resolution spectral studies of brighter GRBs, make EXIST the natural hub and primary trigger of a Next Generation GRB Mission (which might also include a separate x-ray afterglow telescope, as a coordinated small mission, and optical/IR rapid-response capability).

Numerous key questions for the origin and nature of GRBs can be addressed. As but one example, the possible determination of GRB redshifts directly from the GRB data using the possible correlation of GRB luminosity and temporal lags over a broad energy band, as recently suggested by Norris et al [11] (cf. Figure 5) is well suited to the broad band energy coverage and high time resolution of EXIST.
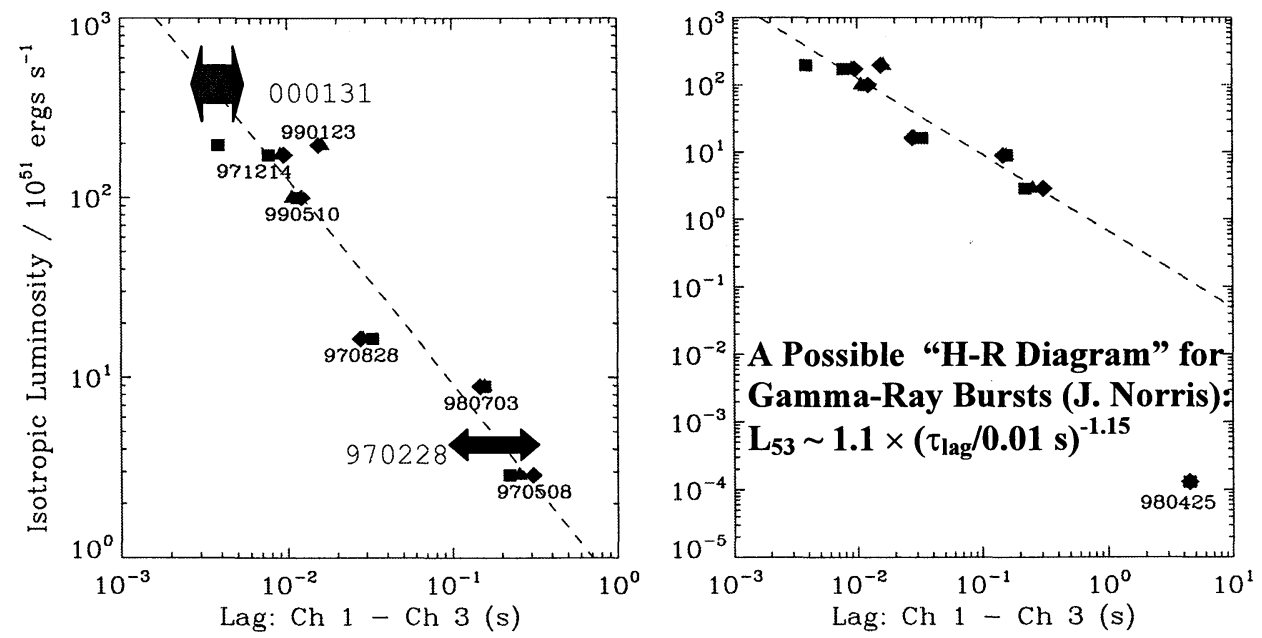

FIGURE 5. Adapted from [11], with 2 bursts added (blue): GRB luminosity vs. measured time lag $(\sim 50-300 \mathrm{keV})$ for BATSE data on GRBs with measured redshifts. The broad energy band and high sensitivity/temporal resolution of EXIST yield such "photometric" redshifts for a large GRB sample. 
EXIST would increase the sensitivity for GRB detection by factors of $\sim 5 \mathrm{X}$ (vs. Swift) and $\sim 20 \mathrm{X}$ (vs. BATSE). As such, it will measure the very faintest GRBs, locate them within $\sim 10 \mathrm{sec}$ to $\leq 1$ ' (GRBs above the BATSE threshold would have positions limited only by the absolute aspect uncertainties, probably $\sim 5 "$ ), and provide high time resolution spectra that can be constructed from the un-binned photon arrival times $(\sim 1 \mu \mathrm{sec}$ resolution) that would be brought down in the full telemetry for followup analysis. With $\sim 60 \%$ of the BATSE instantaneous sky coverage but $\sim 20 \mathrm{X}$ increased sensitivity, the GRB detection rate should be $\sim 3-5 \mathrm{X}$ that of BATSE so that comparable GRB samples (but for much fainter bursts) can be achieved in $\sim 2$ years. The spectral resolution expected for the large CZT array ( 1-3 keV (FWHM) at $\leq 200$ $\mathrm{keV} ; \sim 3-6 \mathrm{keV}$ at higher energies) enables searches for fast-time spectral variations which could constrain fireball and internal shock models as well as the possibility of $511 \mathrm{keV}$ (blue or red-shifted) in the GRB-afterglow transition spectrum.

These capabilities allow EXIST to carry out the most sensitive search for and measurements of GRBs at high redshifts, $\mathrm{z} \sim 5-20$, and thus realize the full potential of GRBs as probes of the high redshift universe [12]. The primary objectives include:

- extending $\log N-\log S$ to the limit, with a large sample of faint GRBs which both maximizes the measurement of the GRB luminosity function (e.g. searches for more 980425 events) and the redshift distribution of GRB sources.

- measurement of PopIII star formation, with GRBs at the putative $\mathrm{z} \sim 10-20$ epoch of PopIII star formation expected if these earliest stars were massive and likely to produce hypernova or collapsar systems as expected [13] for GRBs.

- constrain metallicity at high $z$, since detection of even a moderate sample of high z (>7) GRBs with early ( $<1$ d) afterglow spectra (near and mid-IR) would enable Ly- $\alpha$ forest measures of the history of metallicity enhancement, and growth of structure and galaxy formation, after the initial PopIII starburst era.

\section{Black Holes on All Scales}

Black holes are ubiquitous hard $\mathrm{x}$-ray sources. Both $\mathrm{BHs}$ in galactic $\mathrm{x}$-ray binaries and AGN of all types are detected with a significant fraction of their total luminosity in the $\sim 10-200 \mathrm{keV}$ (rest) band and with spectral distributions remarkably similar [14; cf. Figure 6] for similar source types (BH-LMXBs/Seyfert I and II; microquasars/radio loud QSOs). Thermal Comptonization processes dominate in the former, with spectral breaks and characteristic electron temperatures typically at $\sim 100 \mathrm{keV}$, and non-thermal power laws in the latter. The high-sensitivity and spatial resolution of the EXIST survey would allow spectral measurements of AGN some 30X fainter than the mean OSSE spectrum shown in the lower right panel of Figure 6. Fundamental questions about accretion onto super-massive BHs can be investigated: i) how do Comptonization temperatures and optical depths scale with luminosity? ii) how do Compton reflection components $(\sim 10-30 \mathrm{keV})$ vary in time and on what timescales (for the brigher AGN) which in turn restrict the emission geometry? and iii) how do these same components scale with relativistic-broadened Fe lines from the inner disk which could be measured in detail with simultaneous high resolution observations 
from Constellation-X? New clues to these questions are enabled by the all-sky, alltime measuring/monitoring of AGN possible for the first time with EXIST.
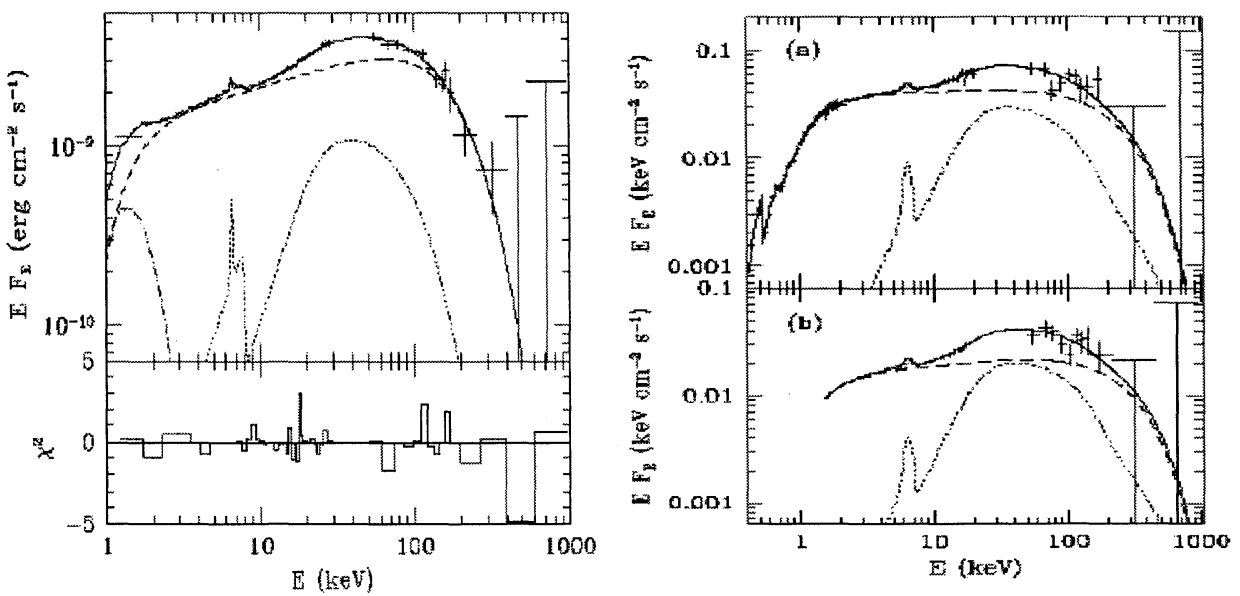

FIGURE 6. Spectra of BH x-ray binary (GX339-4), left, vs. AGN (IC4329A, top; average of 5 Seyfert Is detected by both Ginga and OSSE, bottom), right, showing how similar the thermal Comptonization models (dashed lines) and reflection/fluorescent components (dots) fit both classes (cf. Zdziarski [14]).

Galactic BHs are thus far found predominantly in soft x-ray transients (SXTs) in which the $\mathrm{BH}$ accretes from a low mass secondary companion star when mass transfer is triggered by an ionization instability in the accretion disk. Dynamical masses ( $3-7$ solar masses) have been measured for 13 of these when the secondary star becomes detectable between outbursts, and a very much larger galactic population $(\geq 3000)$ is expected depending on recurrence times. EXIST would detect and locate these SXTs anywhere in the Galaxy for outbursts either $\geq 100 \mathrm{X}$ fainter and/or shorter than typical values $(\sim 100-300 \mathrm{mCrab}, \sim 10-30 \mathrm{~d})$ so that the full population of galactic $\mathrm{BHs}$ in low mass binaries could be studied. BHs accreting from high mass companions must be much more numerous than the one dynamically confirmed case (Cyg X-1); a large population may be lurking among the Be-binary transients for which HX discovery is needed given their expected obscuration in the galactic plane.

Finally, the EXIST survey is uniquely able to find, or limit, the number of possible intermediate mass BHs (with $\geq 30-100 \mathrm{Msun}$ ) which would be detected anywhere in the Galaxy if they pass through giant molecular clouds with typical densities $\geq 10^{3} \mathrm{~cm}^{-3}$ at their expected velocities $(\sim 10 \mathrm{~km} / \mathrm{s})$ by the Bondi-Hoyle accretion expected. Thus, measurement of the expected hard X-ray spectra from isolated black holes offers a unique probe of the $\mathrm{BH}$ content of the Galaxy. If the Milky Way contains $\sim 10^{9}$ neutron stars produced by $\sim 10$ Msun progenitors, and if $\mathrm{BHs}$ form from $>30$ Msun stars, then $\sim 2 \times 10^{8}$ BHs are expected within $\sim 200 \mathrm{pc}$ of the plane and $\sim 10^{3}$ would be within, and accreting from, dense giant molecular clouds to be discovered by EXIST. 


\section{Neutron Stars and SGRs}

Accretion-powered pulsars (e.g. Her X-1), with either low or high mass companions, are readily detected and monitored by EXIST throughout the Galaxy. Thus, the studies initiated with BATSE for pulsar timing and accretion torques [15] can be extended to a much larger neutron star (NS) population of both disk-fed and wind-fed systems, which will include the large population of relatively faint $\mathrm{x}$-ray pulsars with Be companions expected as NS transient systems. The recent BeppoSAX discovery of a significant new population of faint $(\sim 10 \mathrm{mCrab}) \mathrm{NS}$ transients/bursters among the LMXBs [16] suggests a larger reservoir to be discovered with EXIST. These may include more sources like SAX J1808.4-3658, the only known accretionpowered millisecond pulsar and direct link to the millisecond pulsar population. EXIST can measure the magnetic fields for virtually the entire $\mathrm{x}$-ray pulsar population from cyclotron lines at energies $\sim 10 \mathrm{keV} \times \mathrm{B}_{12}$ for fields of strength $\mathrm{B}_{12} \times 10^{12}$ gauss.

Soft Gamma-ray Repeaters (SGRs) are the extremes of the NS population and likely are magnetars with magnetic fields $\geq 100 \mathrm{X}$ that $\left(\sim 10^{12}\right.$ gauss $)$ of ordinary accretion-powered pulsars. Only three are confirmed in the Galaxy, and yet their highly irregular duty cycles for their sporadic $\sim 10 \mathrm{msec}, \sim 30 \mathrm{keV}$ bursts suggests there are many more, which could constrain the evolution of magnetic fields on NSs. The rare super-bursts observed so far from two SGRs (one in the LMC) are so luminous that EXIST could measure them out to the Virgo cluster for the first survey of isolated NSs in external galaxies and possible links to the short-duration GRB population.

\section{Supernova and Nova Rates in the Galaxy}

The death rate of both massive and few solar mass stars can be constrained with EXIST. Type II SNe eject ${ }^{44} \mathrm{Ti}$ from a layer close to the mass cut within which material in the core collapses. The decay of this isotope with 96y half-life is marked by $\mathrm{HX}$ lines at 68 and $78 \mathrm{keV}$, easily resolved by EXIST. The full galactic plane survey of EXIST will reveal the supernova remnants (SNR) totally obscured by dust in the galactic plane. Scaling from the 2 SNR detected by the accompanying $1.18 \mathrm{MeV}$ line (cf. Diehl, these proceedings), EXIST should detect and locate $\left(\leq 2^{\prime}\right)$ all SNR within $\sim 8 \mathrm{kpc}$ for the past $10^{3}$ years, or $\sim 15 \pm 4$ for a nominal SN rate of $\sim 3 /$ century. The observed sample thus measures the galactic SNII rate and can be studied with Con-X.

Stellar novae are expected to be signalled by a flash of $511 \mathrm{keV}$ emission from

${ }^{18} \mathrm{~F}$, which emits positrons as it decays with $158 \mathrm{~min}$ half-life. The scattering of these same photons produces a detectable emission through the entire EXIST energy range, down to X-ray energies. After this initial explosion, which could only be detected with an all-sky $511 \mathrm{keV}$ imager like EXIST, other characteristic line emission from elements like ${ }^{7} \mathrm{Be}$ ( $478 \mathrm{keV}$ energy, half-life of 77 days) can be detected for new measures of the nova rate in the Galaxy and the origin of the diffuse $511 \mathrm{keV}$ emission.

\section{POSSIBLE IMPLEMENTATION OF EXIST CONCEPT}

The very large area imaging CZT array presents the greatest technical challenge for 
the development of EXIST. The detector-telescope packaging concept for an ISS implementation of EXIST is shown in Figure 1 and in more detail in Figure 7. A CZT

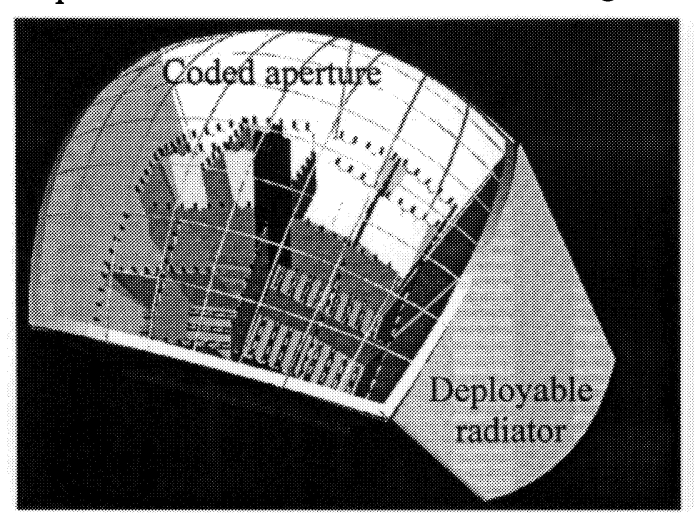

FIGURE 7. Partial detail of concept EXIST-ISS telescope ( 1 of 2 ; cf. Fig. 1) showing CZT arrays $\left(16 \times 2500 \mathrm{~cm}^{2}\right)$, CsI collimators, and extended coded aperture mask and supporting structures.

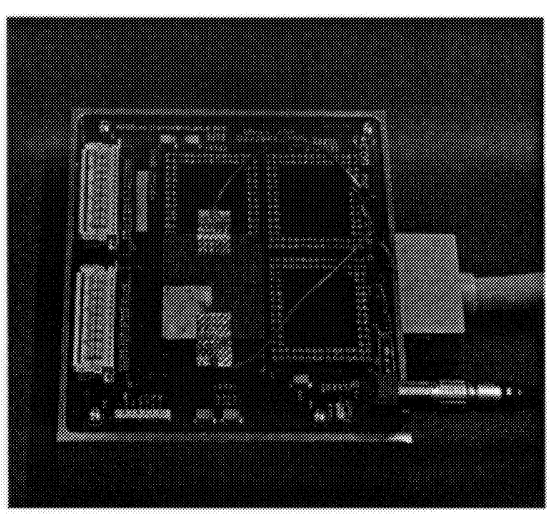

FIGURE 8. Prototype tiled CZT array [17] module $(8 \mathrm{~cm} \times 8 \mathrm{~cm})$ with $2 \times 2$ subarray of $2 \mathrm{~cm} \mathrm{CZT} \mathrm{crystals} \mathrm{with} \mathrm{test} \mathrm{cathode} \mathrm{contacts}$ (3 Au +1 In, showing).

imager development program is underway at 4 of the institutions of the EXIST Science Team (Harvard, GSFC, Caltech and UCSD) and elsewhere. An example prototype module with ASIC readout (2.5mm pixels; 256 channels) on each of 4 close-tiled $4 \mathrm{~cm} \times 4 \mathrm{~cm}$ CZT sub-arrays is shown in Figure 8. An EXIST imager module could be fabricated from scaling this tiling up a factor of 4 to $32 \mathrm{~cm} \times 32 \mathrm{~cm}$.

EXIST-ISS is currently estimated to be $5500 \mathrm{~kg}$ and require $1.5 \mathrm{Mb}$ telemetry and $1.5 \mathrm{~kW}$ power. Design studies for a Free Flyer version of EXIST, as well as a balloonborne demonstration imager (for a ULDB proto-survey mission), are in progress.

\section{REFERENCES}

1. Fabian, A. and Iwasawa, K., MNRAS, 303, L4 (1999).

2. Levine, A. et al, Ap. J. Suppl., 54, 581 (1984).

3. Gehrels, N., B.A.A.S., 31, 1512 (1999).

4. Grindlay, J. et al, Proc. SPIE, 2518, 202 (1995).

5. Caroli, E. et al, Sp. Sci. Rev., 45, 349 (1987).

6. Grindlay, J. et al, AIP Conf. Proc., 510, 784 (2000).

7. Fabian, A., preprint (astro-ph/0103439) (2001).

8. Vignati, P. et al, $A \& A, 349$, L57 (1999).

9. Comastri, A. et al, $M N R A S$, in press (astro-ph/0105525) (2001).

10. Coppi, P. and Aharonian, F., Astroparticle Phys., 11, 35 (1999).

11. Norris, J.P., Marani, G.F. and Bonnell, J. T., Ap. J.,534, 238 (2000).

12. Lamb, D. and Reichert, D., Ap. J., 536, 1 (2000).

13. MacFadyen, A., Woosley, S. and Heger, A., Ap. J., 550, 410 (2001).

14. Zdziarski, A., ASP. Conf. Ser., 161, $16(1999)$.

15. Bildsten, L. et al, Ap. J. Suppl., 113, 367 (1997).

16. In 't Zand, J., preprint (astro-ph/0104299) (2001).

17. Narita, T., Grindlay, J. et al, Proc. SPIE, in press (2001). 